\title{
A comparative analysis of in vitro expansion of natural killer cells of a patient with autoimmune haemolytic anaemia and ovarian cancer with patients with other solid tumours
}

\author{
VIDYASAGAR DEVAPRASAD DEDEEPIYA ${ }^{1}$, HIROSHI TERUNUMA ${ }^{2}$, XUEWEN DENG $^{2}$, \\ SUBRAMANI BASKAR $^{1}$, SADANANDA RAO MANJUNATH ${ }^{1}$, RAJAPPA SENTHILKUMAR ${ }^{1}$, \\ PALANISAMY MURUGAN ${ }^{1}$, PARAMASIVAM THAMARAIKANNAN ${ }^{1}$, THANGAVELU SRINIVASAN ${ }^{1}$, \\ SENTHILKUMAR PREETHY ${ }^{1,3}$ and SAMUEL J.K. ABRAHAM ${ }^{1,4}$ \\ ${ }^{1}$ Nichi-In Centre for Regenerative Medicine, Chennai, India; ${ }^{2}$ Biotherapy Institute of Japan, Tokyo, Japan; \\ ${ }^{3}$ Hope Foundation, Chennai, India; ${ }^{4}$ Yamanashi University - School of Medicine, Chuo, Japan
}

Received August 17, 2011; Accepted November 21, 2011

DOI: $10.3892 / \mathrm{ol} .2011 .498$

\begin{abstract}
The functional profile of natural killer (NK) cells has been reported to be lower in auto-immune haemolytic anaemia (AIHA). In this study, we report a comparative analysis of peripheral blood mononuclear cells (PBMNCs) and the in vitro expansion of NK cells in a patient with AIHA and cancer, with that of other cancer patients without AIHA. PBMNCs and in vitro NK-cell expansion of a 64-year old female patient with ovarian cancer and AIHA was compared with that of four other patients with cancer without AIHA who underwent autologous immune enhancement therapy (AIET). The NK cells were cultured using autologous plasma without feeder layers. The quantities of PBMNCs, NK cells and CD3-CD56+ cells were compared. The average quantity of PBMNCs per $\mathrm{ml}$ in Cases I to $\mathrm{V}$ were 10.71, 39.2, 49.26, 65.16 and $49.33 \times 10^{4}$, respectively, and the average maximum count of NK cells was 3.9, 1730.03, 1824.16, 1058.61 and $761 \times 10^{6}$, respectively. The average percentage of CD3-CD56+ cells in Cases I to $\mathrm{V}$ following in vitro expansion was $1.2,65.7,28.63$, 65.9 and $40 \%$, respectively. In the present study, probably the first in the literature, the in vitro expansion of NK cells was found to be significantly lower in the AIHA patient. Previously, only a lower NK-cell functional profile was reported. Further studies are required to establish the association between AIHA
\end{abstract}

Correspondence to: Dr Samuel J.K. Abraham, Nichi-In Centre for Regenerative Medicine, C 16\&17, Vijaya Health Centre Premises, 175, NSK Salai, Vadapalani, Chennai, Tamil Nadu 600026, India E-mail: drabrahamsj@ybb.ne.jp

Abbreviations: NK, natural killer; AIHA, auto-immune hemolytic anemia; PBMNC, peripheral blood mononuclear cells; AIET, autologous immune enhancement therapy; IP typing, immunophenotyping

Key words: auto-immune hemolytic anemia, natural killer cells, CD3+CD56- cells, red blood cell and natural killer cell antibodies, autologous immune enhancement therapy and NK-cell profile and in vitro expansion, and to find common antibodies between red blood cells (RBCs) and NK cells.

\section{Introduction}

The human immune system acts through innate and acquired immune pathways against cancer and viral infections. The natural killer (NK) cells play a vital role in human innate immunity. Their role in curtailing cancers and viral infections is currently being used as a therapeutic strategy in the form of autologous immune enhancement therapy (AIET), which has been used in clinical practice in several developed nations, including Japan, since the early 1990s and has undergone numerous randomized clinical trials in lung, gastric, ovarian and liver cancers (1-4). Evidence has shown that cancer victims have significantly fewer NK cells in their peripheral blood (PB) than normal age-matched controls $(5,6)$. Numerous auto-immune conditions, including multiple sclerosis, systemic lupus erythematosus and type I (auto-immune) diabetes mellitus, have been reported to be associated with a comparatively lower NK-cell profile (7-10). However, an evaluation of the in vitro expansion of NK cells isolated from the PB of those patients in such conditions has not yet been reported. One such condition where a lower functional profile of NK cells has been reported is auto-immune haemolytic anaemia (AIHA) (11).

In the present study, we report the results of in vitro expansion of NK cells in an ovarian cancer patient who was suggested for AIET using NK cells. However, when we attempted in vitro expansion of NK cells in this patient the quantity of expansion was notably poor and only when the reasons for a lower in vitro expansion were investigated, was a diagnosis of AIHA noted. The subsequent efforts to expand NK cells in vitro from the same patient also yielded poor results. We then performed a comparative analysis of the quantity of peripheral blood mononuclear cells (PBMNCs) and in vitro expansion of NK cells of this patient with another patient with ovarian cancer, without AIHA, as well as with 3 more patients with other types of solid tumours admitted for AIET. Therefore, the present study aimed to present the results found and a thorough discussion on these findings. 


\section{Materials and methods}

Case details. All procedures were carried in accordance with local and national regulatory guidelines. The procedures followed were in accordance with the ethical standards described by the Helsinki Declaration.

In this study, the PBMNC count, subsequent in vitro expansion of cells in the NK-cell flask and the percentage of expression of CD3-CD56+ cells of Case I, diagnosed with ovarian cancer and later with AIHA, was evaluated and compared with that of 4 other patients with a diagnosis of a solid tumour, but without AIHA or any other auto-immune disease, who underwent AIET for at least 3 cycles within a period of 2 months the previous year. One patient had ovarian carcinoma and the remaining 3 patients had various other forms of solid tumours, as described in the following paragraphs. In all 5 patients, the PB was collected following confirmation that their hemoglobin level and blood cell counts were within normal physiological ranges.

Case I. A female patient aged 64 years presented with advanced serous papillary adenocarcinoma of the ovaries with liver metastasis. The patient underwent omentectomy and resection of the liver nodule in the month of January 2011 and completed 6 cycles of chemotherapy with paclitaxel and carboplatin, 3 cycles pre-surgery and 3 cycles post-surgery. The 6 chemotherapy cycles were completed in April 2011. In April 2011, a computed tomography (CT) scan of the whole abdomen revealed non-enhancing lesions in the left lobe of the liver, the largest measuring approximately $2.1 \times 1.8 \mathrm{~cm}$, and a left gastric node. The patient was suggested for radiofrequency ablation and maintenance chemotherapy. In June 2011, the patient also received AIET using NK cells in addition to these therapies. $\mathrm{PB}$ was withdrawn for the NK-cell isolation and expansion procedure. Since the PBMNCs were markedly lower (although the patient had a white blood cell count of 4,600 cells) and the NK-cell expansion was not marked, the patients history was reviewed in order to identify the cause. Incidentally, during that period, the patient received the report from the hospital in Thailand of her diagnosis of AIHA, on suspicion of which she had been administered steroids. The steroid dose consisted of a dose of prednisolone $15 \mathrm{mg}$ /day for $1 \mathrm{month}$, and the dose was suggested to be tapered. A PB specimen was withdrawn again from the patient 1 week later, and again the PBMNC was low and in vitro expansion of NK cells was extremely low.

Case II. A 54-year-old female diagnosed with papillary serous cystadenocarcinoma of the ovary, stage III-C, in April 2009 was administered 3 cycles of intravenous chemotherapy with paclitaxel and carboplatin every 21 days, followed by a staging laparotomy in June 2009. Subsequently, 3 cycles of intraperitoneal chemotherapy with paclitaxel and cisplatin was administered every 21 days. Recurrence was diagnosed in July 2010, following which, besides chemotherapy with doxorubicin hydrochloride and carboplatin, the patient was administered 3 cycles of AIET. This case closely resembles that of Case I in terms of diagnosis and treatment; however, there was no associated AIHA or any other auto-immune disease.

Case III. A 12-year-old male patient diagnosed with adenocarcinoma of the lower oesophagus was administered 3 cycles of
AIET following a single cycle of intravenous chemotherapy using cisplatin for 3 days.

Case IV. A 63-year-old male patient diagnosed with squamous cell bronchiogenic carcinoma was admitted for AIET, and was administered 3 cycles of AIET along with homeopathic treatment.

Case V. A 70-year-old female patient diagnosed with carcinoma of the breast, had undergone mastectomy of the left breast in 2006. In 2011, the patient presented again with a right intramammary nodule with pulmonary metastasis. The patient was admitted for AIET and administered 3 cycles of AIET inbetween chemotherapy cycles.

PBMNC isolation and quantification. PB was collected from all patients after informed consent was obtained in accordance with local regulatory guidelines. For each cycle of AIET, approximately $70 \mathrm{ml}$ of $\mathrm{PB}$ was collected by venipuncture and transported to the National Centre for Research Methods (NCRM) lab within $12 \mathrm{~h}$, without exposure to X-ray screening, under cool preservation at $4-8^{\circ} \mathrm{C}$. A sample was provided for microbiological screening and the PBMNCs were isolated with Lymphoprep $^{\mathrm{TM}}$ (AXIS-SHIELD PoC AS, Oslo, Norway) in a GMP compliant class 10,000 clean-room using ultra high-speed refrigerated centrifuge, as previously reported (12). The PBMNCs were counted in a phase contrast inverted microscope using the trypan blue exclusion method and documented. The PBMNCs per ml of blood was calculated from the total count.

$N K$ cell in vitro expansion and evaluation. The cells were expanded in a GMP compliant cell processing room in a $\mathrm{CO}_{2}$ incubator (Astec, Japan) using the autologous plasma of the patients, which was derived from the PB. The procedure followed was the same as that reported previously and no feeder layers or animal or allogeneic serum were used during the entire procedure (12). The cell expansion was documented in terms of quantity by manual count under an inverted phase contrast microscope at various intervals, and also for immunophenotyping (IP typing) by flow cytometry (BD FACSCalibur ${ }^{\mathrm{TM}}$, BD Biosciences, Franklin Lakes, NJ, USA). The IP typing was carried out for CD3-CD56+ cell evaluation twice. Initially, PBMNCs isolated from the PB were allocated for IP typing prior to commencing the culture expansion of cells, and the expanded NK cells were then allocated for IP typing prior to transfusion when the maximum count was obtained, immediately following which the cells were harvested for transfusion.

\section{Results}

Patient characteristics and in vitro cell expansion. The detailed results of the PBMNC cell counts, cell expansion details and the percentage of CD3-CD56+ cells are shown in Table I. The average quantity of PBMNCs per $\mathrm{ml}$ of PB in Case I was $10.71 \times 10^{4}$ cells vs. $39.2 \times 10^{4}$ in Case II. In other cases, the average quantity of PBMNCs per $\mathrm{ml}$ of $\mathrm{PB}$ was 49.26, 65.16 and $49.33 \times 10^{4}$ cells, respectively. The maximum count of the

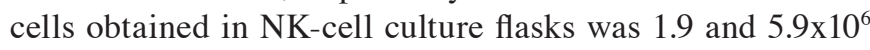
cells (average $3.9 \times 10^{6}$ ) in Case I during 2 consecutive expansions vs. 1483,1676 and $2031.1 \times 10^{6}$ (average $1730.03 \times 10^{6}$ cells) 
Table I. Patient charatcteristics, in vitro cell expansion and characterization details.

\begin{tabular}{|c|c|c|c|c|c|}
\hline Characteristic & Case I & Case II & Case III & Case IV & Case V \\
\hline Age (years) & 64 & 54 & 12 & 61 & 70 \\
\hline Gender & $\mathrm{F}$ & $\mathrm{F}$ & $\mathrm{M}$ & M & $\mathrm{F}$ \\
\hline \multirow[t]{3}{*}{ PB qty (ml) } & 70 & 105 & 100 & 50 & 45 \\
\hline & 35 & 120 & 100 & 50 & 45 \\
\hline & & 120 & 150 & 120 & 45 \\
\hline \multirow[t]{3}{*}{ Per ml MNC (x10) } & 7.14 & 34.28 & 51 & 70 & 49.33 \\
\hline & 14.28 & 41.66 & 51 & 70 & 49.33 \\
\hline & & 41.66 & 45.8 & 55.5 & 49.33 \\
\hline Average $\left(\times 10^{4}\right)$ & 10.71 & 39.2 & 49.26 & 65.16 & 49.33 \\
\hline Initial MNC & 5 & 36 & 51 & 35 & 22.2 \\
\hline \multirow[t]{2}{*}{ seeded $\left(\times 10^{6}\right)$} & 5 & 50 & 51 & 35 & 22.2 \\
\hline & & 50 & 68.7 & 66.6 & 22.2 \\
\hline Max cell count & 1.9 & 1483 & 1810 & 1062 & 675 \\
\hline obtained in NK-cell & 5.9 & 1676 & 1610 & 1041 & 975 \\
\hline flask $\left(\times 10^{6}\right)$ & & 2031.1 & 2052.5 & 1073 & 633 \\
\hline Average $\left(\times 10^{6}\right)$ & 3.9 & 1730.03 & 1824.16 & 1058.66 & 761 \\
\hline Day of harvest/ & 8 & 12 & 10 & 14 & 12 \\
\hline day of max growth & 6 & 12 & 12 & 16 & 14 \\
\hline (day) & & 12 & 12 & 12 & 14 \\
\hline Initial CD3-CD56+ & 8.5 & 3.9 & 4.8 & 9.6 & 8.2 \\
\hline \multirow[t]{2}{*}{$(\%)$} & 0.4 & 7 & 4.8 & 9.6 & 8.2 \\
\hline & & 7 & 2.6 & 0.2 & 8.2 \\
\hline \multirow{3}{*}{$\begin{array}{l}\text { Max CD3-CD56+ } \\
(\%)\end{array}$} & NA & 48.1 & 13 & 56.7 & 72.1 \\
\hline & 1.2 & 76.4 & 31.3 & 62.3 & 29.7 \\
\hline & & 72.6 & 41.6 & 78.7 & 18.2 \\
\hline
\end{tabular}

Data obtained on the patient characteristics, PB qty harvested in each transfusion, qty of PBMNCs obtained, qty of PBMNCs seeded, cell count obtained following expansion in the NK-cell culture flask, the max number of days the cells were grown in culture for each transfusion in Cases I, II, III, IV and V are shown. PBMNC, peripheral blood mononuclear cell; PB, peripheral blood; MNC, mononuclear cell; NK, natural killer; qty, quantity; max, maximum.

in 3 consecutive expansions in Case II. The average counts of 3 expansions in the remaining cases (Cases III, IV and V) were $1824.16,1058.61$ and $761 \times 10^{6}$ cells, respectively. The average percentage of CD3-CD56+ cells on the day of maximum growth or the day of harvesting in Case I following in vitro expansion was $1.2 \%$, while in Case II it was $65.7 \%$; in Case III it was $28.63 \%$, in Case IV it was $65.9 \%$ and in Case V it was $40 \%$. Thus, when the average values of the 4 cases (Cases II, III, IV and V) were maintained as $100 \%$, in Case I the average PBMNC count was $11.7 \%$, the expansion of cells in the NK-cell flask was $0.29 \%$ and the percentage of CD3-CD56+ cells was $2.39 \%$. Fig. 1 shows the flow cytometry images of the PBMNC specimens and that following in vitro expansion.

The difference in PBMNC counts and the range of cell expansion in the NK-cell culture flask between the cases is shown in Figs. 2 and 3. The difference in the percentage of NK cells expressing CD3-CD56+ markers between the 5 cases is shown in Fig. 4. In all the 5 cases, the methodology and techniques of the in vitro expansion procedures, the lot number of the laboratory reagents, handling staff and equipment used were identical.

\section{Discussion}

Results of this study suggest that, along with the decrease in the functional profile of NK cells as described in the literature (7-11), in AIHA, the in vitro expansion of NK cells is also severely impaired; as documented in the AIHA patient with ovarian cancer. The decrease in the expansion of NK cells in the first sample of Case I led to a review of the patient's medical history, which revealed a suspicion of an associated auto-immune disease by the physicians for which the patient was recommended steroids. During the course of the in vitro expansion, the diagnosis of AIHA was confirmed by the tests conducted. Moreover, the consecutive samples of PBMNCs also had a decreased expansion in spite of all procedure-related variables, including the laboratory reagents being equivalent. The effect of corticosteroid administration on NK-cell expansion may be considered to be a limiting factor; however, the steroid dose was tapered prior to the start of the in vitro NK-cell expansion. Therefore, we believe that the effect of steroids on the expansion of NK cells may not be significant in the outcome of decreased expansion. Furthermore, the leukocyte 
A a

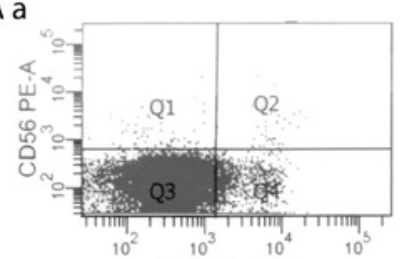

CD3 PerCP-A

B a

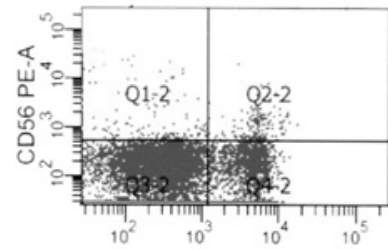

$\mathrm{Ca}$

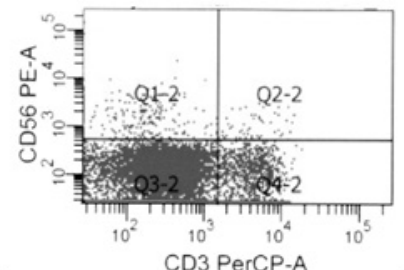

$\mathrm{Da}$

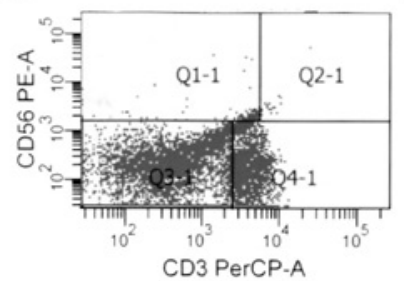

E a

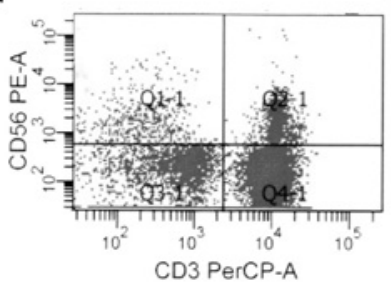

$\mathrm{Ab}$

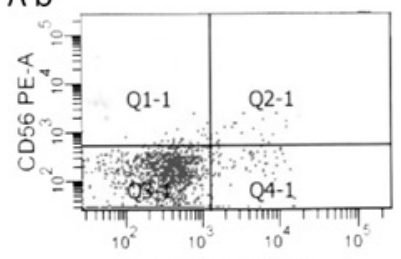

$\mathrm{Bb}$

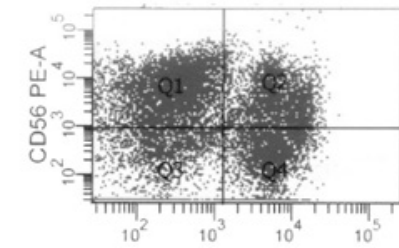

$\mathrm{Cb}$

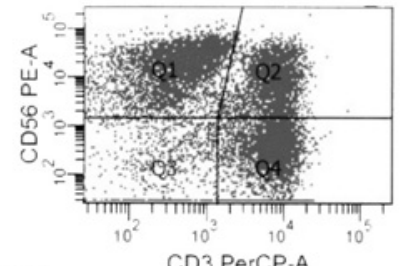

$\mathrm{Db}$

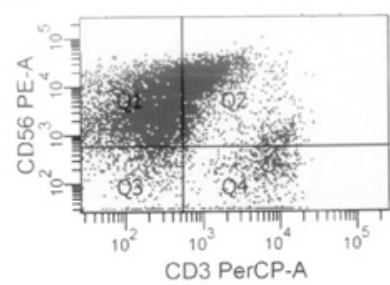

$E b$

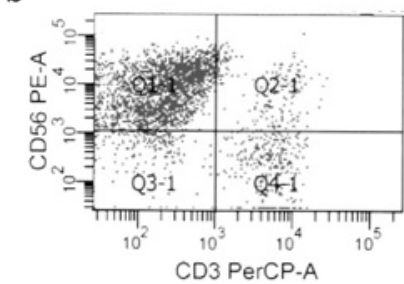

Figure 1. Flow cytometry images of the PBMNC specimens (left panels) and that following in vitro expansion (right panels). CD3-CD56+ cells are visible in the upper left quadrant of each image. (Aa) Case I prior to expansion. (Ab) Case I following expansion. (Ba) Case II prior to expansion. (Bb) Case II following expansion. (Ca) Case III prior to expansion. (Cb) Case III following expansion. (Da) Case IV prior to expansion. (Db) Case IV following expansion. (Ea) Case V prior to expansion. (Eb) Case V following expansion. PBMNC, peripheral blood mononuclear cell.

count was normal prior to collections of PB for NK-cell expansion. When the NK-cell expansion of this case was compared to a similar case of ovarian carcinoma without AIHA or any other auto-immune disease that underwent AIET in the past year following the same protocols and procedures, it was found that the expansion of NK cells was optimal in the ovarian cancer patient without AIHA, in accordance with previous reports $(12,13)$. The same case (Case I) was compared with another 3 patients with other forms of solid tumours, without AIHA or any other auto-immune disease, who had undergone at least 3 cycles of AIET in the previous year. A relatively lower NK-cell expansion in Case I was further confirmed as shown

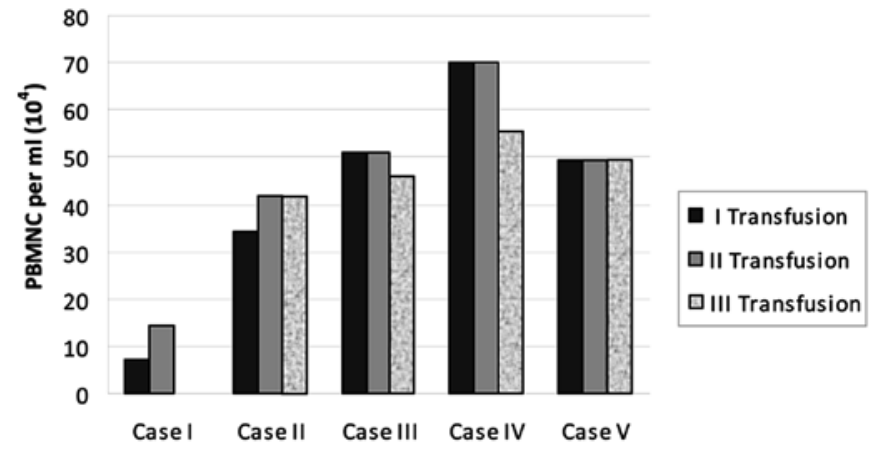

Figure 2. Comparison of the quantity of peripheral blood mononuclear cells (PBMNCs) per $\mathrm{ml}$ of peripheral blood (PB) collected in the consecutive transfusions in all the cases (Cases I, II, III, IV and V).

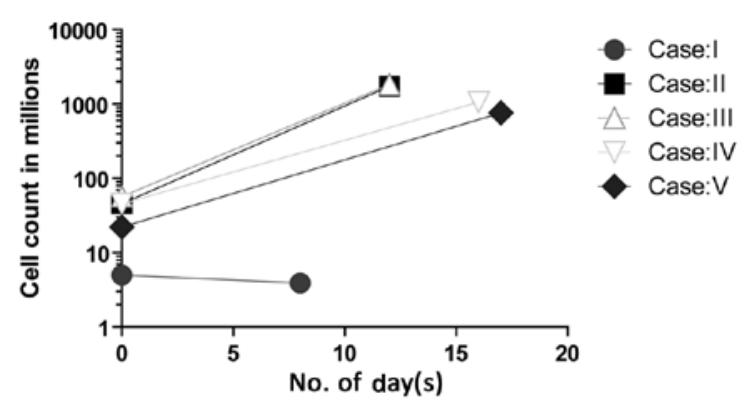

Figure 3. In vitro expansion pattern of the cells in the natural killer (NK) cell culture flask.

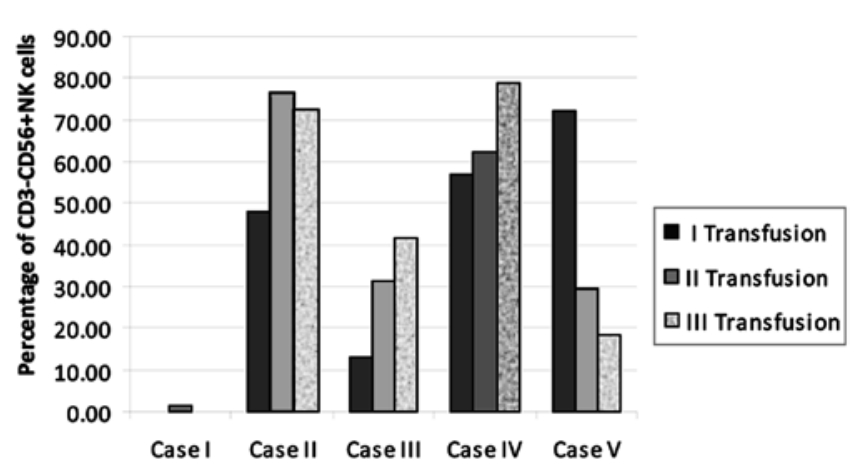

Figure 4. Comparison of the percentage of CD3-CD56+ NK cells in consecutive transfusions in all 5 cases (Cases I, II, III, IV and V). NK, natural killer.

in Figs. 2 and 3. The percentage of CD3-CD56+ NK cells was also decreased in Case I compared with the other cases (Fig. 4).

The association of AIHA with lymphoproliferative disorders and other haematological malignancies as a paraneoplastic phenomenon is well known (14). There have been numerous case reports in the literature documenting the association of AIHA with solid tumours $(15,16)$, particularly ovarian tumours (17-19). The association of AIHA with ovarian tumours is less than $1 \%$ and it has been suggested to include ovarian teratoma as one of the differential diagnoses of AIHA (18).

In an analysis by Puthenparambil et al of 52 cases of solid tumours with AIHA, it was found that curative resection of the associated primary tumours led to complete, and often 


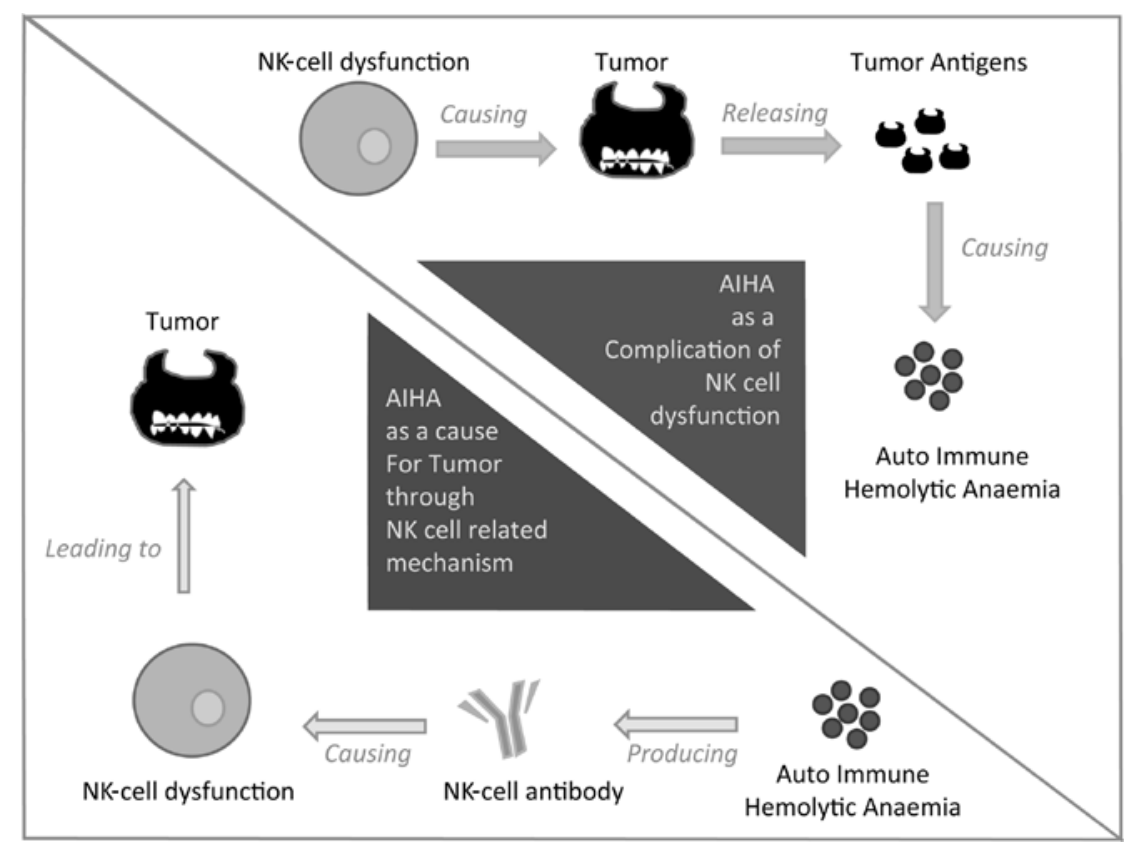

Figure 5. The hypothesis of the association between autoimmune haemolytic anaemia (AIHA), natural killer (NK) cell profiles and tumour formation is shown. The upper right triangle shows the explanation of AIHA as a cause of lower NK-cell profiles and the lower left triangle describes how AIHA may lead to the development of a tumour.

sustained, remission of AIHA (15). In addition, those patients who were refractory to steroid treatment prior to resection of the tumour became responsive following the resection. In another study, it has been reported that AIHA associated with malignant lymphomas or tumours, is eliminated following successful anti-lymphoma or anti-tumour therapy (20). Hoffman et al have postulated that whereas AIHA is a well-known complication of malignant lymphoproliferative disorders; lymphoproliferative disorders paradoxically appear as a consequence of AIHA (14). Based on this statement, AIHA is likely to be the cause and consequence of malignancies, although the former must be substantiated with adequate evidence. Therefore, the role of tumour-related antigens in such well-documented primary malignancies in the development and progression of AIHA when studied may reveal further insights into the mechanisms of action, which enable us to arrive at targeted therapies against those antigens.

While considering the AIHA as a cause of other malignancies, the association of a lower NK-cell profile should be investigated. The study by Imai $e t$ al on an 11-year followup of 3,625 residents of a Japanese community for their immune cell profile indicated that medium and high cytotoxic activity of peripheral-blood lymphocytes is correlated with a decrease in cancer risk, whereas low activity is correlated with an elevated cancer risk (5). In their study, Montelli et al concluded that NK-cell activity is decreased in family members of patients with various types of cancer compared to healthy controls of similar parameters (6). Similarly, the study by Dewan et al revealed that NK activity of PBMNCs is lower in breast cancer patients (21). These findings suggest that a lower NK-cell profile may be considered as a phenomenon or mechanism underlying the development of cancer in general. Further studies should be undertaken to ascertain whether the lower NK-cell profile may have first caused a malignancy, which, in turn, produced antigens, leading to the development of AIHA as a complication (Fig. 5); or whether a lower NK cell itself may be attributed to the development of an AIHA. A lower NK-cell profile has already been reported in patients with AIHA; however, the count of NK cells in those patients with a lower NK-cell profile has been found to be normal (11). Therefore, the mechanism that causes the functional profile and the cytotoxicity to be lower, leading to a hole in the immune surveillance, would be of critical importance. In AIHA, autoantibodies against red blood cells (RBCs) lead to haemolysis, and reports have highlighted the presence of antibodies against other proteins, including actin (22). Investigations into the presence of a common antibody for RBCs and NK cells or the precursors should be undertaken to find the association between AIHA and lower NK cell profiles (Fig. 5) with regard to the cause or complication mechanisms of AIHA to cancers centered on NK cells.

It has also been proven that in vitro expanded NK cells have a 5- to 10-fold increased cytotoxic ability compared to purified blood cells without expansion (23). The most crucial point of concern in this report is the significantly lower NK cell in vitro expansion itself using the autologous serum of this AIHA patient. While in other forms of cancer, the NK cells may have had a lower functional profile when analysed in vitro, their in vitro expansion was optimal, which is in agreement with earlier reports. However, when the same methodology was utilized in this patient it was found to be significantly lower, suggesting that there may have been a common antibody in the serum of the patient that inhibited their in vitro expansion. Efforts to obtain the expansion using an allogeneic serum may resolve this question.

In conclusion, this case report, the first of its type in the literature, has yielded new insight into the extremely lower in vitro expansion ability of NK cells using autologous serum 
in an AIHA patient with ovarian tumour. A larger study involving more AIHA patients is required to verify the findings. A decreased NK-cell profile has been reported in patients with solid tumours as well as in AIHA patients. Although such a decrease of NK cells in solid tumour patients is considered to be a cause for the tumours, in AIHA such a decrease is hypothetically considered to be a complication. Further studies and extensive analysis of the mechanisms underlying these phenomena, particularly the search for a common antibody between RBCs and NK cells or its precursors, are likely to reveal the exact mechanism of these associations.

\section{Acknowledgements}

The authors acknowledge the M/S Hope Foundation (Trust), Chennai, India for providing funding for this study.

\section{References}

1. Goto S, Shirotani N, Hatakeyama M, et al: Clinical benefit of non-toxic therapy in patients with advanced cancer (opinion). Anticancer Res 22: 2461-2464, 2002.

2. Kimura $\mathrm{H}$ and Yamaguchi Y: A phase III randomized study of interleukin-2 lymphokine-activated killer cell immunotherapy combined with chemotherapy or radiotherapy after curative or noncurative resection of primary lung carcinoma. Cancer 80 42-49, 1997

3. Takayama T, Sekine T, Makuuchi M, et al: Adoptive immunotherapy to lower postsurgical recurrence rates of hepatocellular carcinoma: a randomised trial. Lancet 356: 802-807, 2000

4. Kono K, Takahashi A, Ichihara F, et al: Prognostic significance of adoptive immunotherapy with tumor-associated lymphocytes in patients with advanced gastric cancer: a randomized trial. Clin Cancer Res 8: 1767-1771, 2002.

5. Imai K, Matsuyama S, Miyake S, Suga K and Nakachi K: Natural cytotoxic activity of peripheral-blood lymphocytes and cancer incidence an 11-year follow-up study of a general population, Lancet 356: 1795-1799, 2000

6. Montelli TC, Peracoli MT, Gabarra RC, Soares AM and Kurokawa CS: Familial cancer: depressed NK-cell cytotoxicity in healthy and cancer affected members. Arq Neuropsiquiatr 59: 6-10, 2001.

7. Grunebaum E, Malatzky-Goshen E and Shoenfeld Y: Natura killer cells and autoimmunity. Immunol Res 8: 292-304, 1989.
8. Baxter AG and Smyth MJ: The role of NK cells in auto-immune disease. Autoimmunity 35: 1-14, 2002.

9. Cameron AL, Kirby B and Griffiths CE: Circulating natural killer cells in psoriasis. Br J Dermatol 149: 160-164, 2003.

10. French AR and Yokoyama WM: Natural killer cells and autoimmunity. Arthritis Res Ther 6: 8-14, 2004.

11. Conte R, Dinota A, Tazzari PL, Belletti D and Sermasi G: Analysis of natural killer cells in patients with idiopathic autoimmune hemolytic anemia. Vox Sang 56: 270-273, 1989.

12. Takada M, Terunuma H, Deng X, Dewan MZ, Saji S, Kuroi K, Yamamoto $\mathrm{N}$ and Toi M: Refractory lung metastasis from breast cancer treated with multidisciplinary therapy including an immunological approach. Breast Cancer 18: 64-67, 2011.

13. Spanholtz J, Preijers F, Tordoir M, et al: Clinical-grade generation of active NK cells from cord blood hematopoietic progenitor cells for immunotherapy using a closed-system culture process. PLoS One 6: E20740, 2011.

14. Hoffman PC: Immune hemolytic anemia - selected topics. Hematol Am Soc Hematol Educ Program 13-18, 2006.

15. Puthenparambil J, Lechner K and Kornek G: Auto-immune hemolytic anemia as a paraneoplastic phenomenon in solid tumors: a critical analysis of 52 cases reported in the literature. Wien Klin Wochenschr 122: 229-236, 2010.

16. Al-Matham K, Alabed I, Zaidi SZ and Qushmaq KA: Cold agglutinin disease in fibrolamellar hepatocellular carcinoma: a rare association with a rare cancer variant. Ann Saudi Med 31: 197-200, 2011.

17. Morris PG, Swords R, Sukor S, Fortune A, O'Donnell DM and Conneally E: Auto-immune hemolytic anemia associated with ovarian cancer. J Clin Oncol 26: 4993-4995, 2008

18. Kim I, Lee JY, Kwon JH, et al: A case of auto-immune hemolytic anemia associated with an ovarian teratoma. J Korean Med Sci 21: 365-367, 2006.

19. Park SB, Kim JK, Kim KR and Cho KS: Imaging findings of complications and unusual manifestations of ovarian teratomas. Radiographics 28: 969-983, 2008.

20. Valent $P$ and Lechner K: Diagnosis and treatment of auto-immune haemolytic anaemias in adults: a clinical review. Wien Klin Wochenschr 120: 136-151, 2008.

21. Dewan MZ, Takada M, Terunuma H, Deng X, Ahmed S, Yamamoto $\mathrm{N}$ and Toi M: Natural killer activity of peripheralblood mononuclear cells in breast cancer patients. Biomed Pharmacother 63: 703-706, 2009.

22. Felder KM, Hoelzle K, Heinritzi K, Ritzmann M and Hoelzle LE: Antibodies to actin in auto-immune haemolytic anaemia. BMC Vet Res 6: 18, 2010.

23. Terunuma H, Deng X, Dewan Z, Fujimoto S and Yamamoto N: Potential role of NK cells in the induction of immune responses: implications for NK cell-based immunotherapy for cancers and viral infections. Int Rev Immunol 27: 93-110, 2008. 